\title{
Segmenting the Sepal and Shoot Apical Meristem of Arabidopsis thaliana
}

\author{
Alexandre L. Cunha ${ }^{1,2}$, Adrienne H. K. Roeder ${ }^{2,3}$, Elliot M. Meyerowitz ${ }^{3}$
}

\begin{abstract}
We present methods for segmenting the sepal and shoot apical meristem of the Arabidopsis thaliana plant. We propose a mathematical morphology pipeline and a modified numerical scheme for the active contours without edges algorithm to extract the geometry and topology of plant cells imaged using confocal laser scanning microscopy. We demonstrate our methods in typical images used in the studies of cell endoreduplication and hormone transport and show that in practice they produce highly accurate results requiring little human intervention to cope with image aberrations.
\end{abstract}

\section{INTRODUCTION}

The weed Arabidopsis thaliana is the de facto model organism in plant molecular biology and it is one of the most studied plant model systems [1]. In our current investigations we aim to understand how biochemical signaling coupled with mechanical forces and cell geometry governs cell growth and gives rise to patterns in plants. Image segmentation is an important step in these developmental studies as it provides ways to quantify shape, size, and positioning of cells over time.

We are particularly interested in segmenting two regions of Arabidopsis: the sepal and the shoot apical meristem (SAM). The sepal is a defensive organ that encloses and protects the developing reproductive structures. The outer sepal epidermis contains a characteristic pattern of diverse cell sizes ranging from giant cells one-fifth the length of the sepal to small cells one-hundredth the length of the sepal. Shoot apical meristems are collections of stem cells found at the tip of each growing shoot of a plant. They provide the cells that will differentiate into leaves and flowers (including sepals), and into the stem. The SAM is not only an interesting example of a stem cell population that is highly regulated in its size, patterns of cell division, and products, but also is of practical importance, as most of what humans eat (such as grains and fruits) is derived from it.

Previous work. There are a few articles describing segmentation methods for Arabidopsis. In [2], Marcuzzo et al. propose a cell segmentation method for the Arabidopsis root. They apply the watershed method and then discard regions falsely labeled as cells using a SVM classifier which is trained to identify false cells based on a cell contour descriptor. Results show a reduced false-positive rate of true cells as compared to the pure watershed segmentation results. Our work differs from theirs in the segmentation

\footnotetext{
${ }^{1}$ Center for Advanced Computing Research, ${ }^{2}$ Center for Integrative Study of Cell Regulation, ${ }^{3}$ Division of Biology, California Institute of Technology, Pasadena, California 91125. cunha, aroeder, ctobin, meyerowecaltech.edu
}

procedure and we do not discard badly segmented cells but rather offer ways to correct them. The work of de Reuille et al. [3] proposes a protocol to extract cell geometry and topology in the outer surface layer of the Arabidopsis apical meristem. Their method approximates the surface by manually marking junction vertices where cell walls meet and manually assigning which vertices belong to each cell. An automatic procedure then determines the $2 \mathrm{D}$ polygonal cell topology and maps it to the 3D surface of the meristem. Contrary to them, we automatically, or semi-automatically, compute the outline of each cell and then automatically determine junction vertices and overall cell topology. In addition, we allow for curved cell walls.

In the next section we present our segmentation methods followed by a section with results and concluding remarks.

\section{Methods}

We propose segmenting sepals and meristems using the variational formulation of Chan and Vese [4] and a mathematical morphology based pipeline of our own targeted to our images. Both approaches have benefited from robust denoising prior to segmenting. We explain all methods in the following subsections.

Data collection. To measure the epidermal cell size in sepals, the plasma membrane of epidermal cells were fluorescently labeled with pATML1::mCitrine-RCI2A. A confocal laser scanning microscope was used to capture 3D stacks of the sepal epidermis. Images were acquired in vivo at low illumination to avoid damaging the plant tissue. Since the sepal is nearly flat, maximum intensity projections were made. While cell walls in projections are more intense, the overall image contains more spurious data coming from different slices of the stack. Further, the same cell walls in different slices do not completely align sometimes giving the false impression that double parallel walls exist. Generally, a whole sepal could not fit in one single image frame, so multiple frames were taken and manually assembled to obtain an entire sepal image. Meristems were also fluorescently labeled and imaged using a confocal microscope but neither projecting nor mosaicing were necessary.

Notation. An image $I: \mathbb{G} \subset \mathbb{Z}^{*^{2}} \rightarrow \mathbb{Z}^{*}$, for $\mathbb{G}$ an indexed grid, has quantized intensity values $u_{i} \in[0, L]$, e.g. $L=255$, at grid points $\left(x_{i}, y_{i}\right), i=1, \ldots,|I|$. When necessary we refer to $u$, with no subscript, as the intensity $u(x, y) \in \mathbb{R}$ of the continuous image $I: \Omega \subset \mathbb{R}^{2} \rightarrow \mathbb{R}$. $u^{0}$ denotes the intensity of the original, noisy image $I^{0}$. 


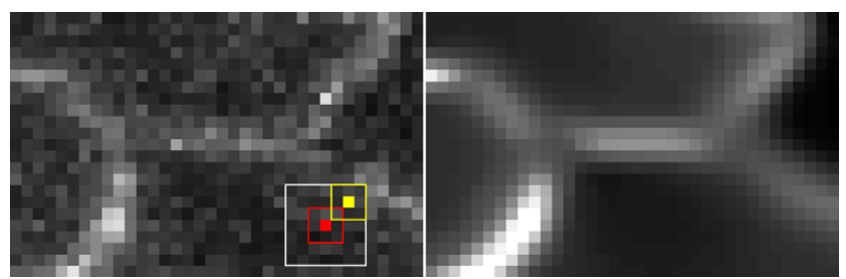

Fig. 1. Denoising improves cell wall localization. Example image on the left shows a magnification of four neighboring meristem cells. Denoising accentuates the localization of their walls thus facilitating posterior segmentation. We used here $m=4$ denoising iterations with $\left|N_{i}^{k}\right|=7 \times 7$ (white square inset), $\left|P_{i}^{k}\right|=3 \times 3$ (red and yellow squares), and $h^{k}=2$, for all iterations $k \in[0,3]$. Histogram stretching was used to enhance the contrast of the denoised image shown on the right.

\section{A. Noise reduction}

Low illumination, necessary to avoid photobleaching and live tissue damage, resulted in significant shot noise in all slices of the confocal $\mathrm{z}$-stack and in its maximum intensity projection. Noise in fluorescence microscopy typically follows a Poisson distribution so it would be natural to adopt a Poisson denoising filter for our plant images. We instead use nonlocal means whose core formulation assumes a Gaussian additive noise. In practice, a scale space nonlocal means behaved extremelly well to separate cell walls from cell interior and background despite not being of Poissonian nature.

We used the fast algorithm and implementation for nonlocal means described in [6] but recasted in a scale space fashion: for each pixel $i \in I$ we compute its $(k+1)$-th restored value $u_{i}^{k+1}$ using the nonlinear convex combination

$$
u_{i}^{k+1}=\sum_{j \in N_{i}^{k}} w_{i j}^{k} u_{j}^{k}, \quad k=0, \ldots, m-1
$$

where the symmetric weights $w_{i j}^{k}=w_{j i}^{k}>0$ form a partition of unit, $\sum_{j} w_{i j}^{k}=1$. The weight $w_{i j}^{k}$ measures how much pixel $j$, located in the immediate neighborhood (square patch centered at $i$ ) $N_{i}^{k}$ of pixel $i$, contributes to the restored value of $i$ at iteration $k$. We compute weights using

$$
w_{i j}^{k}=\frac{1}{W_{i}^{k}} \frac{1}{1+h^{k^{2}} d_{i j}^{k^{2}}}
$$

where $d_{i j}^{k} \equiv d\left(P_{i}^{k}, P_{j}^{k}\right)$ is a measure of similarity between the square patches $P_{i}^{k}$ and $P_{j}^{k}$ centered, respectively, at pixels $i$ and $j$ (see Fig. 1); $h^{k} \in \mathbb{R}^{+}$is a global parameter governing the similarity decay and smoothing $\left(h^{k}=0\right.$ gives the mean filter). In the special case $j=i$ we have $w_{i i}^{k}=\max _{j \neq i}\left\{w_{i j}^{k}\right\}$. High (weak) similarity implies a large (small) positive contribution among pixels. $W_{i}^{k}$ is the normalization coefficient $W_{i}^{k}=\sum_{j}\left(1+h^{k^{2}} d_{i j}^{k^{2}}\right)^{-1}$. Let $\mathbf{v}_{i}^{k} \equiv \mathbf{v}\left(P_{i}^{k}\right)$ be the vector of intensities for pixels in $P_{i}^{k}$, $\mathbf{v}_{i}^{k}=\left\{u_{l}^{k} \mid l \in P_{i}^{k}\right\}$, and $\mathbf{v}_{i j}^{k}=\mathbf{v}_{i}^{k}-\mathbf{v}_{j}^{k}$. Then

$$
d_{i j}^{k}=\left\|\mathbf{v}_{i j}^{k}\right\|_{2}=\left\langle\mathbf{v}_{i j}^{k}, \mathbf{v}_{i j}^{k}\right\rangle^{\frac{1}{2}}
$$

where $\|\cdot\|_{2}$ is the Euclidean $l_{2}$ norm, gives an efficient way of computing similarity between patches. Patches and neighborhoods are typically squares with $P_{i}^{k} \subseteq N_{i}^{k}$ and $\left|P_{i}^{k}\right|=\left|P_{j}^{k}\right|,\left|N_{i}^{k}\right|=\left|N_{j}^{k}\right|$, and their sizes are allowed to change at each iteration $k$ and for every pixel throughout the image.

Computing (1) can be quite expensive: for each pixel $i \in I$ we need to visit all its $\left|N_{i}\right|$ neighbors and for every neighbor compute a distance metric involving $\left|P_{i}\right|$ calculations. The total arithmetic cost per iteration is then $O\left(|I|\left|N_{i}\right|\left|P_{i}\right|\right)$. In [6] this has been reduced to $O\left(|I|\left|N_{i}\right|\right)$. But the communication cost, i.e. moving data between levels of memory hierarchy, is quite more expensive if not carefully addressed. In our current implementation a $1024 x 1024$ image is filtered in little less than 0.3 seconds (wall clock time) for $\left|N_{i}\right|=11 \times 11$ and any size of $P_{i}$. All timing results we report in this article are for runs on a Intel ${ }^{\mathfrak{C}}$ Core $17-965,4$ cores, $3.2 \mathrm{GHz}, 8 \mathrm{MB}$ cache computer. We refer the reader to [6] for details about our fast multicore implementation.

\section{B. Variational segmentation}

The celebrated Active Contours Without Edges segmentation model [4] is attractive due to its simplicity and because it doesn't require image derivatives typically employed to detect region boundaries. And it is specially attractive to our problem because we only have two regions to segment: cell walls and cell interior which have intensities similar to background. The model can be viewed as a thin rubber band with longitudinal stiffness $\mu>0$ that is allowed to bend, break, and join in order to separate the image domain in inside and outside regions, each possibly disconnected and each having, respectively, average intensity of $c_{1}>0$ and $c_{2}>0$.

Let $\phi: \Omega \subset \mathbb{R}^{2} \rightarrow \mathbb{R}$, be a level set function with the usual attributes: $\phi>0$ inside the region of interest, $\phi<0$ outside, and at the interface between these regions $\phi=0$. Following [4], $\phi$ has energy $\mathcal{J}(\phi)$

$\mathcal{J}(\phi)=\int_{\Omega}\left(u-c_{1}\right)^{2} H(\phi)+\left(u-c_{2}\right)^{2}(1-H(\phi))+\mu|\nabla H(\phi)|$

where $H(\phi)$ is the Heaviside function used to localize inside, $H=1$, and outside, $H=0$, regions. The first two terms of $\mathcal{J}(\phi)$ correspond to the data fidelity we want to impose: $c_{1} \rightarrow \bar{u}$ on cell walls and $c_{2} \rightarrow \bar{u}$ inside cells and background ( $\bar{u}$ is the mean value of $u$ ). The last term in (4) gives the length of the rubber that we need to control with a large enough $\mu$ to avoid unnecessary wiggly curves and with a sufficiently small $\mu$ to allow topology changes when necessary but without picking irrelevant tiny regions in the image. Choosing an appropriate $\mu$ does matter to obtain good results and numerical convergence.

After considering Neumann boundary conditions on $\phi$ one obtain the gradient descent Euler-Lagrange PDE equivalent to minimizing $\mathcal{J}(\phi)$

$$
\frac{d \phi}{d t}=\delta_{\varepsilon}(\phi)\left[\mu \kappa(\phi)-\left(u-c_{1}\right)^{2}+\left(u-c_{2}\right)^{2}\right]=0
$$

where $\phi(x, t)$ now depends on the fictitious variable $t, \phi(x, 0)=\phi^{0}, \kappa(\phi)=\nabla \cdot \frac{\nabla \phi}{|\nabla \phi|}$ gives the mean curvature of the surface implied by $\phi$, and $\delta_{\varepsilon}(\phi)=\varepsilon /\left(\pi\left(\varepsilon^{2}+\phi^{2}\right)\right)$ is the $\varepsilon$-mollified delta function. We modified the numerical 


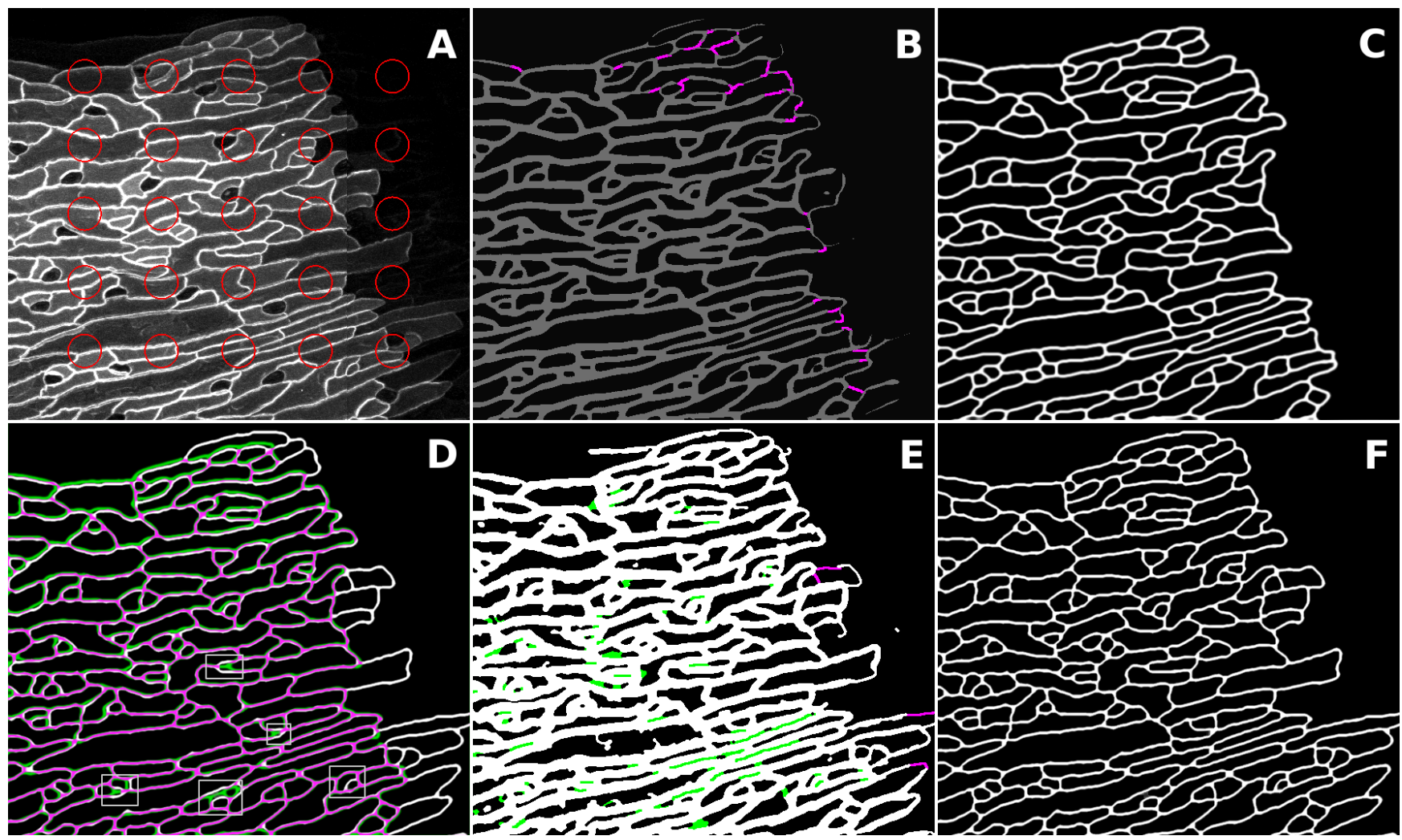

Fig. 2. Sepal cells segmented with active contours and morphological operators. Top row - active contours: a portion of the maximum intensity projection of a sepal confocal 3D-stack is shown in (A) with red circles corresponding to the initial zero level contours, $\phi^{(0)}=0$, used by the variational method. (B) Automatically segmented cell walls are shown in gray color with manually added patches shown in magenta. Most often we need to add other than remove patches when using the variational method. (C) Stylized final cell walls after thinning and pruning the repaired thick edges in (B). Bottom row - morphology: image (E) shows the thick edges obtained by the morphological method and the necessary repairs in green (erase) and magenta (add). Note that in this particular case more editing is necessary when compared to the variational result in (B) but in turn we obtain a larger number of segmented cells. In (F) we have the stylized cell walls after morphological segmentation. In (D) one can appreciate the difference between variational (shown in green) and morphological segmentations (shown in white): regions colored magenta show where cell walls agree in both segmentations. A few boxes in (D) highlight regions where segmentations mostly differ either due to wall localization or completely missing cell walls (not counting whole cells solely present in $(\mathrm{F})$ but not in $(\mathrm{C}))$.

discretization used in [4] to solve (5) as we integrate using explicit, not implicit, finite difference schemes for time and space derivatives of $\phi$. This significantly improves efficiency as we don't need to solve a linear system at each time step. But this comes at a price: the time integration is no longer unconditionally stable. In practice, for this particular problem, the explicit scheme behaves extremely well. Let $\kappa_{\epsilon}(\phi)=\nabla \cdot(\nabla \phi /(|\nabla \phi|+\epsilon))$, where we add $0<\epsilon \ll 1$ to the denominator to avoid division by zero (e.g. $\epsilon=10^{-4}$ ), and $\kappa_{\epsilon}^{(k)}=\kappa_{\epsilon}\left(\phi^{(k)}\right)$ its finite difference approximation. We then obtain updates for $\phi$, starting from a given $\phi^{(0)}$,

$$
\phi^{(k+1)}=\phi^{(k)}+\Delta t \delta_{\varepsilon}^{(k)}\left[\mu \kappa_{\epsilon}^{(k)}+\left(u-c_{2}^{(k)}\right)^{2}-\left(u-c_{1}^{(k)}\right)^{2}\right]
$$

where all the terms on the right hand side are evaluated for $\phi^{(k)}$. Note that $c_{1}$ and $c_{2}$ must be recomputed after each update of $\phi$ as new average values develop when the inside and outside regions are modified.

Since gradient descent can be extremely slow in some cases it is common practice to iterate (6) for a large fixed number of iterations. We instead opt for another convergence criteria. From (5),

$$
\nabla \cdot \frac{\nabla \phi}{|\nabla \phi|}=\frac{1}{\mu}\left[\left(u-c_{1}\right)^{2}-\left(u-c_{2}\right)^{2}\right]
$$

shows, maybe more clearly, that $c_{1}$ and $c_{2}$ are the forces driving the modification of $\phi$, and consequently the segmentation. Thus it is important to track how they change during the numerical integration. Therefore, in addition to a maximum allowed number of iterations, we achieve convergence when $\left|c_{1}^{(k+1)}-c_{1}^{(k)}\right|<\beta$ and $\left|c_{2}^{(k+1)}-c_{2}^{(k)}\right|<\beta$ for some small $\beta$ (e.g. $\beta=10^{-6}$ ). What this means is that we decide to stop whenever the number of pixels exchanging regions is irrelevant to modify both average intensities. At this point there is little to no progress in the propagation of the interface which can be a sign that we converged to a reasonable segmentation or that the chosen value for $\mu$ does not allow further developments. As a safeguard measure, we apply this stopping criteria only after it is repeated a certain number of consecutive times (e.g. 10 times). In practice, we had to learn which values of $\mu$ were suitable across multiple images of sepals and meristems (in general $\mu \in[0.1,3.0]$ ). 


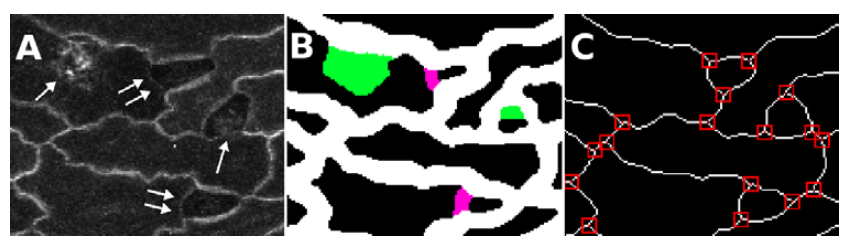

Fig. 3. Interactive editing with a binary brush. (A) A closer look in a region of a sepal image reveals spots where cell wall information is very weak (double arrows) and where contamination appears partly due to the projection procedure (single arrows). Consequently, the thick edges built using the morphology operators are faulty in (B). Contamination is removed by painting with black (showed in green) and missing regions are completed using a white brush (shown in magenta). (C) After corrections we automatically generate one pixel wide edges and compute junctions where cell walls meet (red squares).

\section{Morphological segmentation}

The simplicity, efficiency, and flexibility of mathematical morphology [5] operators encouraged us to investigate another segmentation method less expensive than solving the PDE in Eq. (5). We were also motivated by the denoising results: we noticed that we could carefully turn the denoised gray scale images into binary images without destroying the location of cell walls. The scale space denoising filter was crucial to obtain good binary images much needed by our mathematical morphology pipeline.

Given a denoised image $I^{m}$, we submit it to the following sequence of transformations (image $R$ is updated after each step):

- Edge detection $(\nabla)$. We used a simple first derivative method to detect the edges: $R \leftarrow \theta\left|\nabla I^{m}\right|, \theta=$ scaling factor to create thick edges.

- Threshold $(D)$. The current image is then thresholded to remove as much as possible regions away from the edges, where the gradient is low (within cells and background): $R \leftarrow D \circ R$.

- Hole closing $(C)$. The ridges of the original edges have zero derivative and they need to be closed after thresholding is applied: $R \leftarrow H \circ R$, where $H$ is the operator that sets a pixel to white if at least five of its immediate neighbors are also white.

- Thinning $(T)$. We thin edges to form a pixel wide lines: $R \leftarrow T \circ R$.

- Pruning $(P)$. We finally prune dangling lines and open contours and remove all isolated white pixels: $R \leftarrow$ $P \circ R$. We obtain something like Fig 3C.

- Editing $(E)$. After investigating results, and when necessary, the user patches the segmentation by editing the binary image generated by the hole closing and then reapply thinning and pruning to obtain a new set of complete cells: $R \leftarrow E \circ R$.

The combination of these operations resulted in good quality but not necessarily perfect segmentations. The drawback of neighborhood denoising with averaging schemes like the one shown in (1) is that they don't preserve contrast. After a number of scale space iterations faint edges might completely disappear (see Fig 3). The user usually can quickly detect missing cells after a visual inspection of the segmented image
$R$ above and then act to improve results interactively.

\section{RESULTS}

After applying our variational and morphological methods to segment images of sepals and shoot apical meristems we have found that in practice our morphological approach is more easily adjustable to image variations. Our $\mathrm{C} / \mathrm{C}++$ variational engine segments a 256x256 image in approximately less than 30s. The MATLAB based morphological method consumes only around $5 \mathrm{~s}$ for the same image. Controlling the key parameters $\mu, \beta, \phi^{(0)}, \Delta t$ in the variational method was more challenging and time consuming but results were visually competitive when tuned values were in place. Both methods often generate partial results only. Coping with all possible image variability and aberrations seems infeasible and automatic results are usually sub-optimal across diverse segmentation algorithms. We advocate for a semi-automatic scheme where the computer provides automatic results that can be easily repaired by human intervention if necessary. We offer users an editing mechanism for painting with a binary brush not the original, high value images but rather a byproduct of the segmentation process (thick edges, see Figure 3).

Results after segmenting 3,378 cells in 12 sepals using the morphological method showed that the user had to paint, in average, less than $3 \%$ of the pixels on the faulty thick edges resulting in high quality segmentations and cell measurements necessary to quantify studies of cell endoreduplication and patterning [7]. For comparison, this same interactive approach was also applied to results obtained with the variational model (see figure 2) and in meristems. We will systematically quantify in the future how much manual editing is necessary in these cases but we expect, in general, similar behavior.

We use the precision-recall framework [8][9] to compare automatic, semi-automatic, and ground-truth segmentation results. Precision $p$ gives the percentage of contour pixels in the source segmentation for which we have a corresponding contour pixel in the target segmentation. The reverse gives the recall $r$ value: the proportion of pixels in the target segmentation that were correctly captured in the source segmentation. The harmonic mean of precision and recall gives the $F$-measure, $F=2 p r /(p+r)$, a single number that indicates how similar two segmentations are. In the ideal case, $p=r=F=1$, i.e. source and target segmentations perfectly match (within some allowed uncertainty). By definition, precision is sensitive to over segmentation while recall is sensitive to under segmentation but $F$ is unbiased. When computing pixel correspondence in two segmentations we consider a small uncertainty in the position of matching pixels to account for imprecision in boundary detection (the same edge in the two segmentations might be off by a few pixels). This is realized by a uncertainty search radius $s$, measured in pixels. A large radius yields high precision and recall values so it is reasonable to limit it to moderate values, e.g. $s \in[2,7]$. We use the fast matching algorithm and 

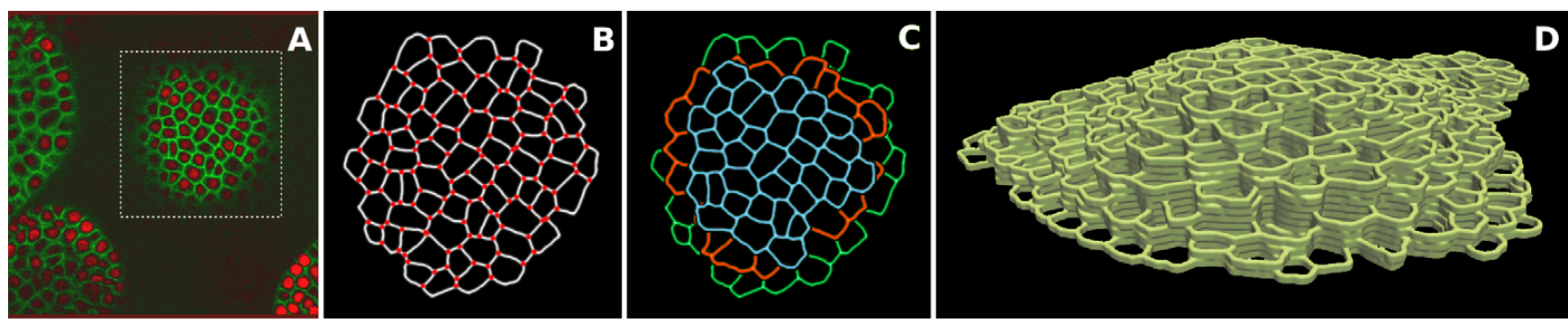

Fig. 4. Illustration of morphological segmentation of meristem cells. The picture in (A) shows one slice of a 3D confocal stack of a shoot apical meristem with the region of interest in the dashed square. Cell plasma membranes are fluorescently labeled with green and nuclei with red. We are interested in segmenting the cell walls and thus we work with the green channel only. In (B) we show our automatic morphological segmentation result (no editing is necessary) with red dots at the junction points. We superimpose the segmentation of three consecutive slices to show in (C) that segmented cell contours largely agree when we move from one slice to the next in the stack, specially for slices close to the epidermis. In (D) we have a 3D visualization of the segmented meristem stack in its natural, live aspect ratio. Manual editing was necessary in some slices to produce this 3D reconstruction, most notably in less illuminated slices deeper in the tissue.

TABLE I

MEAN PRECISION, RECALL, AND F-MEASURE FOR INCREASING RADIUS

\begin{tabular}{c|cccccc|c}
\hline radius & 2 & 3 & 4 & 5 & 6 & 7 & $\mathbf{4 . 5}$ \\
\hline precision & 0.92 & 0.93 & 0.94 & 0.95 & 0.96 & 0.96 & $\mathbf{0 . 9 4}$ \\
\hline recall & 0.84 & 0.85 & 0.86 & 0.87 & 0.87 & 0.88 & $\mathbf{0 . 8 6}$ \\
\hline F-measure & 0.88 & 0.89 & 0.90 & 0.90 & 0.91 & 0.91 & $\mathbf{0 . 9 0}$ \\
\hline
\end{tabular}

TABLE II

GROUND TRUTH PRECISION-RECALL VALUES FOR RADIUS $s=5$

\begin{tabular}{c|ccc|ccc}
\hline \multirow{2}{*}{ sepal \# } & \multicolumn{3}{|c|}{ automatic } & \multicolumn{3}{c}{ semi-automatic } \\
\cline { 2 - 7 } & $p$ & $r$ & $F$ & $p$ & $r$ & $F$ \\
\hline 1 & 0.99 & 0.85 & $\mathbf{0 . 9 1}$ & 0.99 & 0.92 & $\mathbf{0 . 9 6}$ \\
8 & 0.91 & 0.92 & $\mathbf{0 . 9 1}$ & 0.95 & 0.95 & $\mathbf{0 . 9 5}$ \\
15 & 0.89 & 0.93 & $\mathbf{0 . 9 1}$ & 0.96 & 0.95 & $\mathbf{0 . 9 6}$ \\
\hline
\end{tabular}

implementation of [9] to compute precision and recall values for a pair of segmentations.

We compare our automatic to our semi-automatic morphological results (Table I - the last column gives the average over all radii ) and these to ground truth (manual segmentation) results (Table II). In the first case we have for 12 sepals an average $F$-measure of $90 \%$ indicating again that minor editing is necessary to correct the automatic segmentation. The morphological semi-automatic segmentation in turn is very similar to the ground truth: the $F$-measure, as shown in Table II, for three casually chosen and manually segmented sepals gives an accuracy of over $95 \%$ when considering a uncertainty radius of 5 pixels. Our automatic segmentation in this case achieves $91 \%$ of accuracy, which we consider a good success rate.

\section{CONCLUSION}

We presented a numerical scheme for the Chan-Vese variational model and a mathematical morphology based pipeline to segment sepal and meristem cells in the Arabidopsis thaliana plant. Both methods greatly benefit from applying a state-of-the-art denoising filter prior to segmentation. As reported by a precision-recall analysis, our assisted segmentation approach can achieve, in average, over $95 \%$ accuracy when compared to ground truth, manually segmented data. According to our experiments and given the limitations of computer vision in offering fully automatic and completely robust solutions to problems easily corrected by humans we see interactive, semi-automatic approaches similar to ours as compelling mechanisms to deliver quick results to help accelerate biological studies.

\section{ACKNOWLEDGMENTS}

This research was supported by a grant to the California Institute of Technology from the Gordon and Betty Moore Foundation, by Department of Energy Office of Basic Energy Sciences, Division of Chemical Sciences, Geosciences and Biosciences grant DE-FG02-88ER13873, and by National Science Foundation grant IOS-0846192. We thank Marcus Heisler from EMBL Heidelberg for providing confocal images of shoot apical meristems and members of the Computable Plant and Computational Morphodynamics projects at Caltech and UCI for valuable discussions and feedback.

\section{REFERENCES}

[1] E.M. Meyerowitz and R.E. Pruitt, "Arabidopsis thaliana and plant molecular genetics," Science, , no. 229, pp. 1214-1218, 1985.

[2] M. Marcuzzo, P. Quelhas, A. Campilho, A.M. Mendonça, and A.C. Campilho, "Automatic cell segmentation from confocal microscopy images of the Arabidopsis root," in IEEE International Symposium on Biomedical Imaging, Paris, France, May 2008, pp. 712-715.

[3] P.B. de Reuille, I. Bohn-Courseau, C. Godin, and J. Traas, "A protocol to analyse cellular dynamics during plant development," Plant Journal, vol. 44, no. 6, pp. 1045-1053, December 2005.

[4] T.F. Chan and L.A. Vese, "Active contours without edges," IEEE Transactions on Image Processing, vol. 10, no. 2, pp. 266-277, February 2001.

[5] P. Soille, Morphological Image Analysis. Principles and Applications, Springer, 2nd edition, 2004.

[6] J. Darbon, A. Cunha, T.F. Chan, S. Osher, and G.J. Jensen, "Fast nonlocal filtering applied to electron cryomicroscopy," in IEEE International Symposium on Biomedical Imaging, Paris, France, May 2008, pp. 1331-1334.

[7] A.H.K. Roeder, V. Chickarmane, A. Cunha, B. Obara, B.S. Manjunath, and E.M. Meyerowitz, "Variability in the control of cell division underlies sepal epidermal patterning in Arabidopsis thaliana," PLoS Biology, vol. 8, no. 5, 2010.

[8] D. Martin, C. Fowlkes, D. Tal, and J. Malik, "A database of human segmented natural images and its application to evaluating segmentation algorithms and measuring ecological statistics," in IEEE ICCV, 2001, pp. 416-425.

[9] F.J. Estrada and A.D. Jepson, "Benchmarking image segmentation algorithms," International Journal of Computer Vision, vol. 85, pp. 167-181, 2009. 